Editorial: Taking an Indigenous Approach to Study Organizational Behavior in China

\author{
Weichun Zhu \\ School of Labor and Employment Relations \\ The Pennsylvania State University \\ University Park, PA 16802 \\ U.S.A. \\ Email: wzhu@psu.edu \\ Hongwei He \\ Marketing Department \\ University of Strathclyde Business School \\ SWD 507, Glasgow, Scotland, G4 0GE \\ United Kingdom \\ Email: hongwei.he@strath.ac.uk \\ Kenneth S. Law \\ Department of Management \\ The Chinese University of Hong Kong \\ Shatin, N.T., Hong Kong \\ China \\ Email:. mnlaw@cuhk.edu.hk \\ Jiing-Lih Farh \\ Department of Management \\ The Hong Kong University of Science and Technology \\ Clear Water Bay, Kowloon, Hong Kong \\ China \\ Email: $\underline{\text { mnlfarh@ust.edu.hk }}$
}




\section{Editorial: Taking an Indigenous Approach to Study Organizational Behavior in China}

Since the late Chinese leader Deng Xiaoping's "Reform and Open Policy" started in 1978, China's economy has been growing rapidly, and today is the second largest economy in the world. In the globalization age, China's economy has become more integrated and increasingly interdependent with the rest of the world. In this historical transformative moment, practitioners and academics alike have shown growing interests in management and organizational behavioral (OB) issues in the Chinese context. For example, we have seen an increasing number of OB studies using Chinese samples published in international OB and management journals each year. Although these OB studies have undoubtedly shed light on the uniqueness and complexity of $\mathrm{OB}$ issues in China, most of these studies tend to rely heavily on Western OB theories and paradigms in testing their proposed hypotheses and make little reference to the contextual factors or indigenous theorization process (Jia, You, \& $\mathrm{Du}, 2011)$. Thus, there is still very limited evidence to suggest that these OB theories developed in the Western contexts are fully aligned with traditional Chinese culture and history, and current economic, social, and cultural developmental stages.

The need for contextualized OB research has been promulgated for more than a decade. Rousseau and Fried (2001) cited two reasons for the importance of contextualization of $\mathrm{OB}$ research: the internationalization of the $\mathrm{OB}$ research domain and the diversification of work and work settings in different cultures. Tsui (2004) made a distinction between contextembedded research and context-specific indigenous research based on the degree of contextualization. Whereas context-embedded research is "context-sensitive" and explicitly models contextual factors as either main effects or as moderators, the indigenous research goes beyond testing an existing theory to use scientific methods to study local phenomena by using local language, local subjects, and locally meaningful constructs (Tsui, 2004, 2006). Jia et al. (2011) have recently evaluated 259 articles published in six leading general 
management and organization journals between 1981 and 2010, which use the Chinese context for their theoretical contributions to management and organization research, and found few of them showing serious attention to context in terms of theorizing and measurement. Thus, there is a strong need for contextualized OB research in the Chinese context.

\section{Themes and Contributions}

This special issue collected five papers (i.e., Chen, Chen, Zhang, Son, Zhang, \& Liu, 2015; Chen, Leung, Li, \& Ou, 2015; Vogel, Mitchell, Tepper, Restubog, Hu, Hua, \& Huang, 2015; Zhang, Long, Wu, \& Huang, 2015; Zheng, Zhu, Zhao, \& Zhang, 2015) that advance the field of contextualized OB research in the Chinese context. These papers can be categorized into four themes according to the various extent of indigenousness or contextualization: indigenous conceptualization and measurement development of a significant phenomenon; theoretical advancement of an indigenous construct; critical application of Western OB concepts/theories in the Chinese context with a contextualized variable; and cross-cultural comparison. These four themes can be seen as a guideline (although not an exhaustive list) that can categorize different ways of conducting contextualized OB research in China.

Indigenous conceptualization and measurement development research refers to the process of identifying a significant common and global phenomenon and then developing a context-specific conceptualization and measurement scale for this phenomenon in the Chinese context. Zheng et al.'s (2015) paper exemplifies this type of contextualized research. They focus on the important phenomenon of employee well-being. As they have argued, employee well-being is becoming an increasingly important issue in China due to its rapid social and economic changes and transformations, the associated work and life stress, and the central theme of social harmonious development in China. Organizations in China have 
begun to realize the important role of employee well-being in enhancing employee productivity and firm performance, and therefore are keen to launch various programs and initiatives to maintain and enhance employee well-being. However, little is known in the literature about what this increasingly important phenomenon (i.e., employee well-being) really means or represents in Chinese organizations. This is further complicated by the presence of multiple types of conceptualization and the absence of consensus even in Western literature. Against this background, Zheng et al. (2015) argue for the need to take an indigenous approach to conceptualize and develop a scale for measuring employee wellbeing in the Chinese context. Through a serious of both qualitative and quantitative studies, they found that employee well-being in Chinese organizations consists of life well-being (LWB), workplace well-being (WWB), and psychological well-being (PWB). An 18-item employee well-being scale has been further developed and tested against its initial reliability and validity. This scale has been successful in predicting employees' job satisfaction, affective commitment, turnover intention, and job performance. In addition, they also applied the scale to the U.S. context, and found that the factor structure of this newly developed scale can be applicable even in the U.S. context, although the loading values of some items differ somewhat across two cultures (i.e., configural invariance). Doing so, Zheng et al.'s (2015) study contributes to the literature in a number of ways. First, their study advances the literature on $\mathrm{OB}$ in China by indigenously conceptualizing an increasingly significant issue in China (i.e., employee well-being) and developing a scale for measuring it. This new scale reflects the unique influence of Chinese culture on the people's perception of well-being in China, although they also noted that that the structural dimensions of their employee wellbeing scale are consistent with Page and Vella-Brodrick's (2009) proposed theoretical model of employee well-being. In this sense, their study does not only advance the field of employee well-being in China but also globally. Second, their study also tries to link the East 
and the West together by testing their measure scale using a U.S. sample and comparing it cross-culturally, which contributes to the existing employee well-being theories in crosscultural contexts.

Theoretical advancement of an indigenous construct refers to the research effort in developing contextualized research by studying a focal existing indigenous phenomenon and examining its role within an existing theoretical framework or in developing a new conceptual model. Both Chen, Leung et al. (2015) and Chen, Chen et al. (2015) can be categorized under this theme. Chen, Leung et al. (2015) focuses on dualistic model of interpersonal harmony, which is an indigenous Chinese construct that has been developed based on the classical Confucian doctrines (Leung \& Brew, 2009; Leung, Brew, Zhang, \& Zhang, 2011). The proposed model differentiates two harmony motives regarding how individuals manage their interpersonal relationships. Harmony enhancement orients people towards active and productive handling of disagreements and conflicts for genuinely harmonious and mutually beneficial interpersonal relationships. In contrast, disintegration avoidance is an instrumental and self-serving motive to avoid the negative consequences of relationship disintegration, and it captures the secular view of harmony notion commonly ascribed to Chinese people that emphasizes conformity and conflict avoidance. Drawing upon the social regulation framework and the conceptualization of these two harmony motives, Chen, Leung et al. (2015) developed a theoretical model to account for the opposite effects of the two motives on creativity via creative effort. Specifically, creative effort has been found to mediate the positive relationship between harmony enhancement and creativity and the negative relationship between disintegration avoidance and creativity. In addition, guided by the person-situation interactionist view and situational strength theory, this research explores reward for creativity as a strong situational factor that attenuates the effects of the two harmony motives on creativity mediated by creative effort. These hypotheses were supported 
by two field studies conducted in China. The most important contribution of this research is related to the finding that the two different harmony motives can have opposing effects on creativity. Identifying creative effort as the mediation mechanism of the harmony motives' effect on creativity reflects the different propensities of the two harmony motives to promote or suppress active and productive problem solving when conflicting views and ideas are encountered. Thus this research interestingly demonstrated that the emphasis of harmony in Chinese culture can both promote and obstruct creativity. The identification of the different moderating effects of reward for creativity on the relationship between harmony motives and creativity also helps to further understand how the proposed mechanisms can explain opposite effects of the two harmony motives. In sum, this paper explores how a Chinese OB issue (i.e., the emphasis on interpersonal harmony in Chinese culture) affects creativity of Chinese employees, thus enriching the understanding of the antecedents of employee creativity in the Chinese context. Finally, the hypothesized model of this paper is developed based on the integration of indigenous theorization and OB theories developed in Western contexts, which exemplifies the potential contribution of Chinese indigenous theorization to universal theorizing.

Chen, Chen et al. (2015) took a "context specific" indigenous approach to study leadermember relationships in the Chinese context. They argue that the current LMX theory does not capture all the aspects of leader-member relationships from a Chinese perspective and leader-member relationship research should explore both etic (universal or transferable) and emic (culture-specific) aspects of those relationships (Rockstuhl, Dulebohn, Ang, \& Shore, 2012). They found that leader-member personal life inclusion (LMG-P), a dimension of leader-member guanxi (LMG) and defined as the extent to which leaders and members include each other in the private domain, can spill over to affect subordinates' contextual performance (i.e., interpersonal facilitation, and job dedication) in the work domain; 
furthermore, this effect was moderated by subordinates' horizontal collectivism orientation, such that LMG-P spilled over to affect contextual performance only for those who were low in horizontal collectivism orientation. At the group level, the variance of LMG-P within a group, referred to as LMG-P differentiation, was related negatively to group performance when the supervisors held a low horizontal collectivism orientation. At the cross level, LMGP differentiation moderated the relationship between LMG-P and job dedication, such that the relationship was positive only when LMG-P differentiation was low.

Chen, Chen et al. (2015) have made significant theoretical contributions to both emic leader-member guanxi theory and etic LMX theory. First, they found that LMG-P, as a unique aspect of leader-member relationships in China, explains additional variance in interpersonal facilitation and job dedication, above and beyond the effects of LMX. Thus, it is critical to study leader-member relationships in Chinese organizations using both the universal (i.e., LMX) and indigenous aspects (i.e., LMG-P) to examine the effects of leadermember relationships on work outcomes. By adopting a boundary spillover perspective through the lens of role expansion and role differentiation, their research also bridges the LMX and LMG theories. Second, their study highlighted the boundary conditions of social exchange spillover from the private domain to the work domain, thus deepening the understanding of the relationship between LMG-P and contextual performance. Their findings showed that the effects of LMG-P on work outcomes are contingent upon contextual factors, such as the degree of LMG-P differentiation. Third, they studied LMG from a multilevel perspective by taking an initiative to explore the relationship between group-level LMG, i.e., LMG-P differentiation and group performance. Chen, Chen et al. (2015) considered both the dyadic LMG-P and LMG-P differentiation at the group level, which leads to a more nuanced understanding of the complexities of the LMG phenomenon. 
Critical application of Western $O B$ concepts/theories in the Chinese contexts with a contextualized variable involves applying established theories (often developed in the Western context but generally applicable globally) as well as indigenous contextual variables often as the moderating factors to explain OB/HRM phenomena in the Chinese context. Zhang et al.'s (2015) paper falls under this category. Their focal research question is: when is pay for performance (i.e. PFP) related to employee creativity? In China, as Zhang et al. (2015) noted, PFP was originally adopted by the reformists to boost productivity in Chinese firms and enhance pay equity by reducing the influence of traditional practices such as guanxi (Tsui $\&$ Farh, 1997). The western theories they employ are cognitive evaluation theory and organizational justice theory. By incorporating the indigenous variable of guanxi HRM practice (defined as the extent to which HR decisions are perceived to be influenced by personal relationships rather than rules and regulations in an organization), they develop a contextualized theoretical model that explains when PFP affects employee intrinsic motivation and creativity in the Chinese context. As noted by the authors, adding this contextualized variable is particularly relevant, since the extant literature offers mixed findings regarding the impact of PFP on employees' intrinsic motivation and creativity. Drawing on the development of cognitive evaluation theory, they argue that employees respond to individual PFP incentives positively only when they trust that the management will administer the overall HR system fairly; therefore they introduce guanxi HRM practice to the model, as it has a strong influence on employees' trust in management. Specifically, they argue that guanxi HRM practice nullifies the positive effect of PFP on employee intrinsic motivation and creativity through reducing employees' trust in their management, because guanxi HRM practice counteracts the equity principle underlying PFP. Their findings were consistently supported by two independent samples from Mainland China and Taiwan. Based on the findings, the authors conclude that the most effective way for Chinese firms to 
implement PFP is to truly break away from the particularistic cultural tradition and fully embrace the equity principles of modern HR practices. In sum, their study helps advance the Chinese OB theory regarding the reward-creativity relationship from a traditional culture perspective.

Cross-cultural comparison research refers to applying a base theoretical framework (often developed in the Western context, but not necessarily always the case) across samples from different cultures (typically Western vs. Eastern cultures) to compare the similarities and differences in the relationships among the focal variables in the general theoretical framework. Often, cultural theories and associated constructs are implicitly (often used as a theoretical justification) or explicitly (often used as moderating variables) applied. Vogel et al. (2015) is an example of cross-cultural comparison research on Chinese OB issues. They focus on the phenomenon of abusive supervision, challenging the strength of the Westernrooted assumption that all hostile supervisory behaviors are perceived and reacted to similarly by employees. Based on the idea that culture shapes the heuristic used by subordinates to assess the fairness of interpersonal interactions, the authors propose a theoretical model that explains cross-cultural differences in the strength of the negative effects of abusive supervision. Comparing Western with Confucian Asian subordinates, the authors claim to find that abusive supervision is less strongly related to subordinates' trust and effort in Chinese culture because this behavior is perceived as less unfair by these subordinates. They replicated their results in a second study and extended their theoretical model by arguing and supporting that individual differences in power distance orientation mediates this complex effect, offering a succinct and interesting explanation. Their study provides contributions to the understanding of abusive leadership and trust in the Chinese context and also between the Chinese and the Western cultures. First, their work provides evidence to support that, unlike more positive leadership behaviors (e.g., transformational, charismatic) which tend to have 
similar effects across cultures, abusive supervision's effect on employees can be more culturally and contextually different. Second, their study compares both the between- and within-culture effects of abusive supervision. Another contribution, as noted by the authors, is that their work raises the possibility that the primarily used measure of abusive supervision may not be entirely appropriate for research in every culture (i.e. measurement equivalence). They further suggest that researchers interested in studying abusive supervision should attempt to understand the norms of a given culture prior to engaging in their research. One point that needs to be noted is that people's understanding of some of the items of this scale might not be the same across different cultures, which raises the necessity of examining the measurement equivalence of a scale before conducting valid cross-cultural comparison analysis (Vandenberg \& Lance, 2000; Van de Schoot, Lugtig \& Hox, 2012).

In addition to Vogel et al. (2015), Zheng et al. (2015) also has a cross-cultural comparison element, which is demonstrated by the inclusion of a U.S. sample in their final study. Zheng et al.'s (2015) work shows that conceptualization and measurement development in the Chinese context could have implications for theoretical advancement of OB theories in other parts of the world. This might suggest that indigenous OB research in China could have a global reach and make significant contributions to the advancement of OB theories in the Western contexts and thus to the development of OB theories in general.

In sum, this special issue makes a number of significant contributions to the literature of OB in China. First, it demonstrates that contextualized OB research in China can be conducted in four different ways: indigenous conceptualization and measurement development of a significant phenomenon; theoretical advancement of an indigenous construct; critical application of Western OB concepts/theories in the Chinese context with a contextualized variable; and cross-cultural comparison of an existing theoretical model. The papers collected in this special issue can be categorized under at least one of these four 
themes. Second, a wide range of important OB issues in the Chinese context are explored in this special issue, including employee well-being, interpersonal harmony, abusive supervision, leader-member relationship, and pay for performance. Third, with regard to the impact of these phenomena and practices, a wide range of employee outcomes are investigated, such as employee creativity, job performance, contextual performance, affective commitment, employee effort, and group performance. In explaining these effects, a wide range of theories (e.g., fairness/justice/equity theories; situational strength theory, cultural orientation theories; social exchange theories) are applied to carefully select the mediation variables and moderating variables.

\section{Future Research Directions}

Despite the collective contributions made by the five papers in this special issue, there are still many unanswered questions that matter to developing OB theories in the Chinese context. It is not our intent to provide an exhaustive list of future research questions; rather we try to list some important topics that could be addressed sooner rather than later in future indigenous Chinese OB research. First, future research can explore the underlying philosophical, cultural, social, economic, political, and media factors that shape OB in Chinese organizations. One important and interesting question is to explore how several different philosophical values, including Confucianism and Zhongyong (Confucius, 2006), Daoism and Legalism (Hucker, 1995), and Socialism and Capitalism (Yang, 2012), independently and jointly interact to influence the managerial and employee behaviors in Chinese organizations.

Second, in addition to examining the effects of traditional philosophical values mentioned above, it is also of importance to explore how historical and contemporary changes and developments, including the revolution, market economy, reform and open policy, western civilization, information age, globalization, and peasant worker movement 
(nong-min-gong, 农民卫), independently and jointly interact to co-influence Chinese managers' and employees' values, norms, patterns, motivations, behaviors and expectations. China's "one-child policy" started almost at the same time as China's "Reform and Open Policy" in 1970s. Chinese people sometimes talk about the phenomenon of "little emperors", which refers to fact that some children who are the only child in their family might have been spoiled by their parents and grandparents. Therefore, it is interesting to explore if there is any difference in the work values and behaviors between employees who are the only child in their family, and those who are not. In addition, it is of research and practical value to investigate how peasant workers (nong-min-gong, 农民工) adapt to the life and work in their firms, and whether and how their work attitudes and behaviors differ from others.

Next, future research might examine if there are any generational differences in the values, beliefs, work attitudes, and behaviors among Chinese employees, such as whether and how those who grew up with the "Cultural Revolution" experience differ from those who grew up in the age of "Reform and Open Policy." Research attempting to find the answers to these questions can make significant theoretical contributions, as well as offer important practical implications to managers and organizations. For example, using the study findings, managers and organizations can design and develop motivation and reward, as well as punishment, strategies and substantive tactics, to motivate employees of different generations and with different family backgrounds.

Further, more work needs to be done regarding indigenous conceptualization, theorization, and measurement developments, in fields such as leadership styles, ethics, emotional regulation, psychological contract, and organizational communications in Chinese organizations. For example, it is of both theoretical and practical value to explore questions such as which Chinese cultural values, norms or beliefs are relevant to specific emotional display and regulation in Chinese organizations, how the functioning and performance of 
work teams (e.g., shared leadership) in Chinese or Asian organizations differ from in Western organizations, and what unique factors (e.g., organizational culture or national culture) account for those differences.

Finally, the Chinese context is complex and can be influenced by different political systems (Mainland China, Hong Kong, Taiwan), different stages of economic development, the extent of economic integration with the rest of the world, and different types of organizational ownerships (e.g., state-owned, western-owned, joint ventures, privatelyowned). So, it would be interesting to explore the within-Chinese cultural differences (i.e., Mainland China vs. Taiwan or Hong Kong, Taiwan vs. Hong Kong) in employees' work values, attitudes, and behaviors. Further, even within Mainland China, researchers might examine whether there are any differences in OB variables, including values, communication styles, and emotional regulation behaviors among different regions (East coast vs. Inlands), and what factors would account for such differences. Last, but not least, with the increasing integration of the Chinese economy with the rest of the world, an increasing number of Chinese expatriates work in many parts of the world. It is of both theoretical value and practical significance to explore how they adapt to the work and life environment in other cultures and countries, and what factors affect their job performances in those contexts.

\section{Conclusion}

Together, the five articles in this special issue have successfully taken an indigenous approach at different levels of contextualized-ness to examine organizational behavior in Chinese context. Although these articles have addressed a wide range of organizational behavioral issues in China, there are still many more issues to be explored as noted above. Therefore, in this respect, taking an indigenous approach to study organizational behavior in China is only just beginning; we believe that there should be enough fields for researchers to plow for many years to come. 


\section{References}

Chen, Y., Chen, Z., Zhong, L., Son, J., Zhang, X., \& Liu, Z. (2015, in press). Social exchange spillover in Leader-member relations: A multi-level model. Journal of Organizational Behavior.

Chen, T., Leung, K., Li. F., \& Ou, Z. (2015, in press). Interpersonal harmony and creativity in China. Journal of Organizational Behavior.

Confucius. (2006). Original Chinese Title [Confucian Analects] In Y. Zhang (Ed.), (Original work published during the Warring States period). Beijing, China: Zhonghua Book.

Hucker, C. O. (1995). China's imperial past: An introduction to Chinese history and culture. Stanford, CA: Stanford University Press.

Jia, L., You, S., \& Du, Y. (2011). Chinese context and theoretical contributions to management and organization research: A three-decade review. Management and Organization Review, 8,173-209.

Leung, K. \& Brew, F. P. (2009). A cultural analysis of harmony and conflict: Toward an integrative model of conflict styles. In R. S. Wyer, C. Y. Chiu, \& Y. Y. Hong (Eds.), Understanding culture: Theory, research and application (pp. 411-428). New York: Psychology Press.

Leung, K., Brew, F. P., Zhang, Z. X., \& Zhang, Y. (2011). Harmony and conflict: A crosscultural investigation in China and Australia. Journal of Cross-Cultural Psychology, $42,795-816$.

Page, K. M., \& Vella-Brodrick, D. A. (2009). The "what," "why" and "how" of employee well-being: A new model. Social Indicators Research, 90(3), 441-458.

Rockstuhl, T., Dulebohn, J. H., Ang, S., \& Shore, L. M. (2012). Leader-member exchange (LMX) and culture: A meta-analysis of correlates of LMX across 23 countries. Journal of Applied Psychology, 97(6), 1097-1130. 
Rousseau, D. M., \& Fried, Y. (2001). Location, location, location: Contextualizing organizational research. Journal of Organizational Behavior, 22(1), 1-13.

Tsui, A. S. (2004). Contributing to global management knowledge: A case for high quality indigenous research. Asia Pacific Journal of Management, 21(4), 491-513.

Tsui, A. S. (2006). Contextualization in Chinese management research. Management and Organization Review, 2(1), 1-13.

Tsui, A. S., \& Farh, J. L. L. (1997). Where Guanxi Matters Relational Demography and Guanxi in the Chinese Context. Work and Occupations, 24(1), 56-79.

Vandenberg, R. J., \& Lance, C. E. (2000). A review and synthesis of the measurement invariance literature: Suggestions, practices, and recommendations for organizational research. Organizational Research Methods, 3(1), 4-70.

Van de Schoot, R., Lugtig, P., \& Hox, J. (2012). A checklist for testing measurement invariance. European Journal of Developmental Psychology, 9(4), 486-492.

Vogel R. M., Mitchell M. S., Tepper B. J., Restubog S. L. D., Hu C., Hua W., \& Huang J. C. (2015, in press). A cross-cultural examination of subordinates' perceptions of and reactions to abusive supervision. Journal of Organizational Behavior.

Yang, B. (2012). Confucianism, socialism, and capitalism: A comparison of cultural ideologies and implied managerial philosophies and practices in the P. R. China. Human Resource Management Review, 22, 165-178.

Zhang, Y., Long, L., Wu, T., \& Huang, X. (2015, in press), When is pay for performance related to employee creativity in the Chinese context? The role of guanxi, HRM practice, trust in management, and intrinsic motivation. Journal of Organizational Behavior. 
Zheng X., Zhu W., Zhao H., \& Zhang, C. (2015, in press). Employee well-being in organizations: Theoretical model, scale development, and cross-cultural validation. Journal of Organizational Behavior. 